\title{
AGO Recommendations for the Diagnosis and Treatment of Patients with Advanced and Metastatic Breast Cancer: Update 2014
}

\author{
Volker Hanfa Florian Schütz ${ }^{b}$ Cornelia Liedtke ${ }^{c}$ Marc Thill ${ }^{d}$ \\ on behalf of the AGO Breast Committee
}

${ }^{a}$ Frauenklinik, Klinikum Fürth; bUniversitätsfrauenklinik Heidelberg; ${ }^{c} K l i n i k$ für Frauenheilkunde und Geburtshilfe, Universitätsklinikum Schleswig-Holstein/Campus Lübeck; 'Klinik für Gynäkologie und Geburtshilfe, Agaplesion Markus Krankenhaus, Frankfurt/M., Germany

\author{
Keywords \\ Guidelines - Locoregional relapse - Metastatic breast \\ cancer - Targeted therapy
}

\section{Introduction}

For the last 13 years, the Breast Committee of the Arbeitsgemeinschaft Gynäkologische Onkologie (German Gynecological Oncology Group, AGO) has issued annually updated evidence-based recommendations for the diagnosis and treatment of patients with early and metastatic breast cancer. The AGO Breast Committee consists of 45 gynecological oncologists specialized in breast cancer and interdisciplinary members specialized in pathology, radiological diagnostics, medical oncology, and radiotherapy. Each update is performed/assembled in accordance with documented rules, by thoroughly reviewing and scoring the recent publications

Members of the 'AGO Breast Committee' in alphabetical order: UteSusan Albert, Marburg; Ingo Bauerfeind, Landshut; Joachim Bischoff, Magdeburg; Jens Uwe Blohmer, Berlin; Klaus Brunnert, Osnabrück; Peter Dall, Lüneburg; Ingo J. Diel, Mannheim; Tanja Fehm, Düsseldorf; Nikos Fersis, Bayreuth; Michael Friedrich, Krefeld; Kay Friedrichs, Hamburg; Bernd Gerber, Rostock; Volker Hanf, Fürth; Nadia Harbeck, München; Jens Huober, Ulm; Christian Jackisch, Offenbach; Wolfgang Janni, Ulm (Co-Chair); Hans H. Kreipe, Hannover (DGP); Thorsten Kühn, Esslingen; Sherko Kümmel, Essen; Cornelia Liedtke, Lübeck; Sibylle Loibl, Neu-Isenburg; Hans-Joachim Lück, Hannover; Michael Lux, Erlangen; Nicolai Maass, Aachen; Gunter von Minckwitz, NeuIsenburg; Volker Möbus, Frankfurt; Christoph Mundhenke, Kiel; Volkmar Müller, Hamburg; Ulrike Nitz, Mönchengladbach; Mahdi Rezai, Düsseldorf; Achim Rody, Lübeck; Anton Scharl, Amberg (Chair); Rita Schmutzler, Köln; Marcus Schmidt, Mainz; Andreas Schneeweiss, Heidelberg (AIO); Ingrid Schreer, Hamburg (DGS); Florian Schütz, Heidelberg; Peter Sinn, Heidelberg (Pathologie); Erich F. Solomayer, Homburg; Rainer Souchon, Tübingen (ARO); Elmar Stickeler, Freiburg; Marc Thill, Frankfurt; Christoph Thomssen, Halle (Saale); Michael Untch, Berlin. chapter by chapter for their scientific validity (Oxford Level of Evidence, LoE; www.cebm.net [1]) and clinical relevance (AGO grades of recommendation; table 1). All AGO Breast Committee members have declared their potential conflicts of interest. Here, we present the 2014 update of these guidelines, focusing on relevant changes introduced this year. The full version of the updated 2014 slide set is available online as a PDF file [2], in English and German. Moreover, a version for patients is also available at www.ago-online.de.

\section{Bone Health and Osteo-Oncology}

The role of bisphosphonates (BP) in the treatment of hypercalcemia $(\mathrm{LoE} 1 \mathrm{a} / \mathrm{A} /++)$, the reduction of skeletal events $(\mathrm{LoE} 1 \mathrm{a} / \mathrm{A} /++)$ in M1oss patients (= patients with 1 bone metastasis), the prevention of antitumor therapy-induced bone loss/osteoporosis ( $\mathrm{LoE} 1 \mathrm{~b} / \mathrm{B} /++)$, and in the reduction of bone pain $(\mathrm{LoE} 1 \mathrm{a} / \mathrm{A} /++)$ is well established. The same is true for denosumab. This antibody causes less nephrotoxicity and less gastrointestinal toxicity, but higher rates of hypocalcemia than BPs. Both denosumab and BPs can cause osteonecrosis of the jaw (ONJ): in the 3-4-weekly treatment of patients in the metastatic setting ONJ has been reported to occur in $1-2 \%$ of cases, whereas in the less intense adjuvant setting ONJ is diagnosed only in a rare number of cases $(<<1 \%)$. The lowest incidences are reported for oral BPs.

The most controversial issue is the use of BPs in adjuvant therapy of patients with breast cancer, where denosumab is still under investigation.

One of the highlights of the 2013 San Antonio Breast Cancer Symposium was the presentation of a meta-analysis of the prospective randomized trials evaluating the effect of BPs on survival $\mathrm{N}$ as part of the adjuvant treatment of patients with early breast cancer. Coleman [3] presented the analysis done by the Early Breast Cancer Trialists' Collabora-

\section{KARGER}

Fax +497614520714

Information@Karger.com

www.karger.com (c) 2014 S. Karger GmbH, Freiburg

$1661-3791 / 14 / 0093-0202 \$ 39.50 / 0$

Accessible online at:

www.karger.com/brc
Prof. Dr. Volker Hanf

\section{Frauenklinik}

Klinikum Fürth

Jakob-Henle-Straße 1, 90766 Fürth, Germany

volker.hanf@klinikum-fuerth.de 
Table 1. AGO grades of recommendation

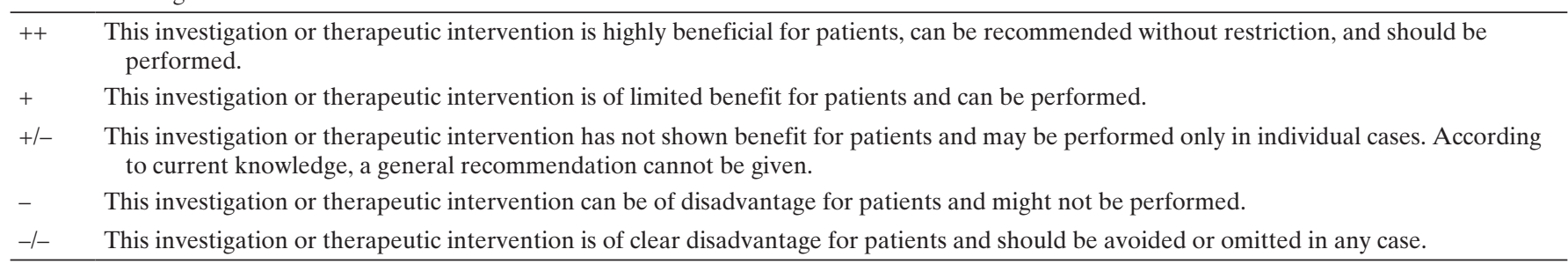

tive Group. Original data from 22,982 patients who were initially randomized in adjuvant treatment protokolls testing BPs versus nihil were assessable. Primary endpoints of these trials were either survival or bone density. 36 trials were identified, 7 using clodronate and 29 using aminobisphosphonates. Data from 17,709 patients were evaluable, comprising $98 \%$ ( $\mathrm{n}=5,053 / 5,174)$ of patients entered into clodronate and $72 \%$ $(\mathrm{n}=12,738 / 17,808)$ entered into aminobisphosphonate trials (zoledronic acid intravenously (i.v.; 65\%), oral ibandronate ( $24 \%)$, or oral pamidronate ( $8 \%$ of the study patients) Primary endpoints were time to first recurrence, time to first distant failure, and overall survival (OS). Recurrence rates were $25.4 \%$ in the BP arm versus $26.5 \%$ for non-BP patients. Distant recurrences occurred in $20.9 \%$ (BP) and $22.3 \%$ (non-BP), respectively. The corresponding event rates for bone metastases were $6.9 \%$ versus $8.4 \%$. In the subset of postmenopausal women (induced menopause or $>55$ years if unknown), distant recurrences were observed in $18.4 \%$ (BP) versus $21.9 \%$ (non-BP) $(\mathrm{p}=0.0003)$, bone metastases in $5.9 \%$ versus $8.8 \%(\mathrm{p}<0.00001)$ and non-bone metastases in $13.3 \%$ versus $14.3 \%$ (not significant). Both the 10 -year breast cancer mortality rate $(18.3 \%$ vs. $15.2 \%, p=0.004)$ and the overall mortality rate $(21.5 \%$ vs. $23.8 \%, p=0.007)$ was significantly reduced by the use of BPs. The results were identical in the clodronate and the aminobisphosphonate group, whether given for cancer or for osteoporosis $(n=4,373)$. Differences were not significant if premenopausal women were included in the analysis.

Based on these data the AGO recommendations have been adjusted, since this meta-analysis shows for the first time a significant 10 -year OS benefit in the range of $3 \%$ due to postoperative treatment with BPs in postmenopausal women (LoE1a/A/+). This benefit is larger than the one observed after the introduction of aromatase inhibitors (AIs) (vs. tamoxifen) or taxanes (taxane/antracyclines vs. antracyclines). Unconditional recommendation $(++)$ was not given due to the heterogeneity of the study population, the regimen, and of the treatment duration.

\section{Follow-Up of Breast Cancer}

It has to be acknowledged that expectations and objectives of follow-up are differentially reported by health professionals or patients.
The main objective of following patients after the primary treatment of breast cancer is the detection of potentially curable events, particularly the detection of local recurrences and contralateral initially breast cancer. With increasing complexity and time length of primary adjuvant treatment, surveillance and counselling during the follow-up period becomes increasingly important. The psychosocial aspects of support and counselling will gain relevance as more patients survive breast cancer and will encounter long-term treatment.

Routine follow-up examinations in asymptomatic patients should comprise history (for specific symptoms), physical examination, mammography, ultrasound of the breast, magnetic resonance imaging (MRI) of the breast in case of inconclusive conventional imaging, and pelvic examination. Breast selfexamination is encouraged by experts especially for selfawareness, but no survival benefit has so far been scientifically substantiated.

Additional examination modalities or shorter examination intervals are explicitly discouraged for the time being, since although intensified surveillance has proved to decrease the lead time until the detection of distant metastases in comparison to conventional surveillance, it has not been shown to improve OS. In the context of novel treatment modalities for distant disease, however, this objective should be reexamined. There are indications that physical activity (as for example walking or yoga) and weight reduction during follow-up can improve quality of life and physical performance, reduce fatigue, and optimize outcome. Therefore, during follow-up, patients should be encouraged to take and supported in keeping up measures to achieve these goals. Intervention in order to treat comorbidities and to counsel for lifestyle risks is recommended in order to reduce unfavorable effects on the course of the breast cancer disease.

During follow-up, compliance with endocrine therapies should be monitored. Predictors for the discontinuation of treatment are young age and old age, breast-conserving therapy (vs. mastectomy), more than 2 comorbidities, higher copayment required, smaller blister pack, and prescription by general practitioners. Predictors of good compliance are marriage, adjuvant chemotherapy, and adjuvant radiotherapy.

Very limited data have been reported in this context and none has been considered to justify a change in treatment recommendation. It has to be emphasized that based on current evidence, the AGO discourages additional follow-up 
examinations in asymptomatic patients, but encourages the performance of future studies on the relevance of additional tests in the context of modern imaging and treatment modalities.

\section{Locoregional Recurrence}

About 10\% (2-20) of patients who undergo breast-conserving surgery and radiation therapy will subsequently develop ipsilateral breast tumor recurrence. Chest wall recurrences after mastectomy and isolated axillary recurrences are relatively rare events. Although the local outcome following salvage therapy is favorable in the majority of cases, the risk of distant metastases for patients with local recurrence is 3-5 times greater than for those without recurrence. The reason for this association has been controversially discussed. However, it now has become an acknowledged fact that local recurrence is both a marker of the underlying biological aggressiveness of the tumor and a possible source for further tumor dissemination.

Only few relevant new data have been reported over the past 12 months. Saigal et al. [4] could show that inflammatory breast cancer at primary diagnosis correlated with increased risk of locoregional recurrence. A meta-analysis reported by Wang et al. [5] confirmed earlier data that triple-negative and/ or human epidermal growth factor receptor 2 (HER2)-positive breast cancer is also associated with increased locoregional recurrence risk. The hazard ratio was 1.88 (1.58-2.22) for triple-negative breast cancer (TNBC) as compared to any other HER2-negative subtypes. However, in comparison to HER2-positive breast cancer, TNBC showed a lower risk with an hazard ratio of $0.69(0.53-0.91)$.

Complete resection of the locally recurrent tumor is the treatment of choice whenever possible. Radiotherapy is needed if there has been no prior irradiation. Hormone receptor (HR)-positive isolated locoregional recurrences

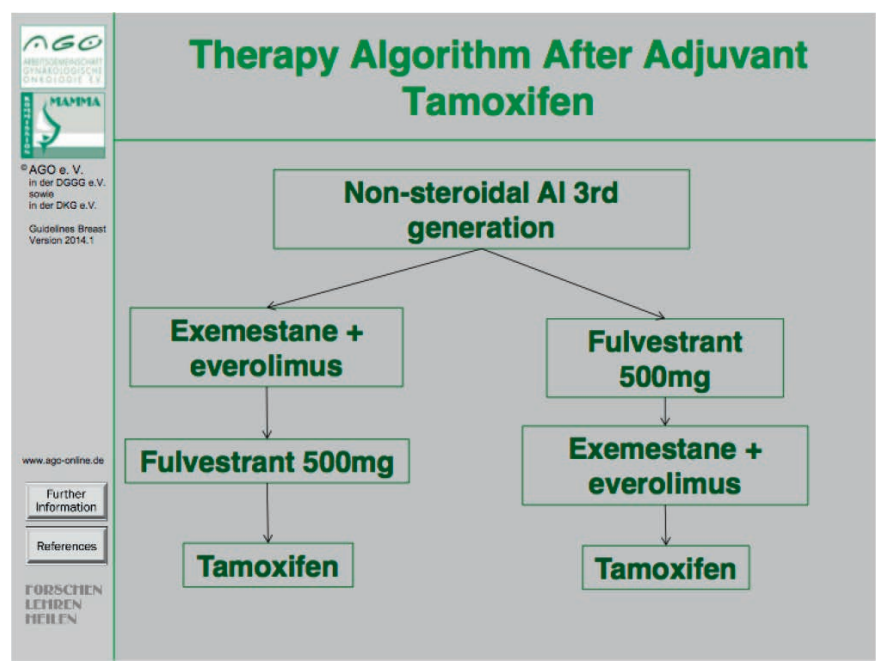

Fig. 1. Therapy algorithm after adjuvant tamoxifen treatment. should receive endocrine treatment. HR-negative patients should receive an effective adjuvant-analogue chemotherapeutic regimen. In case of HER2 positivity, a HER2-targeting procedure should be added, since isolated locoregional recurrence offers the last chance for breast cancer cure. New radiotherapy data published by Skinner et al. [6] showed no benefit of moderate dose escalation (+10\%) after mastectomy. So far, the standard dose regimen remains the radiotreatment of choice. Based on this limited novel evidence, no relevant changes of the AGO recommendations have been made.

\section{Endocrine and Targeted Therapy in Metastatic Breast Cancer}

Endocrine therapy is the backbone of and first choice for the treatment of HR-positive metastatic breast cancer. Therefore, metastatic lesions should be reanalyzed by biopsy and immunohistological staining, if possible. However, even if the analysis may show persisting HR positivity, the tumor cells may have generated resistance against endocrine treatment.

In peri- and premenopausal patients, the induction of ovarian function suppression (gonadotropin-releasing hormone $(\mathrm{GnRH})$ analogue or luteinizing hormone ( $\mathrm{LH})$ analogue) is the first step in endocrine treatment. If possible, it should be combined with tamoxifen $20 \mathrm{mg} /$ day [7]. An AI can be used if there are contraindications against tamoxifen. However, monotherapy with ovarian function suppression or tamoxifen alone is also an option.

In postmenopausal patients, endocrine treatment should be selected depending on previous medication and time line. The first choice of endocrine treatment should be an AI if either tamoxifen has already been used in the primary setting (fig. 1) or an AI treatment was terminated more than 1 year before [8]. If the cancer progressed on therapy with non-steroidal AI (letrozole or anastrozole) or if an AI therapy was terminated less than 1 year ago, two distinct options for endo-

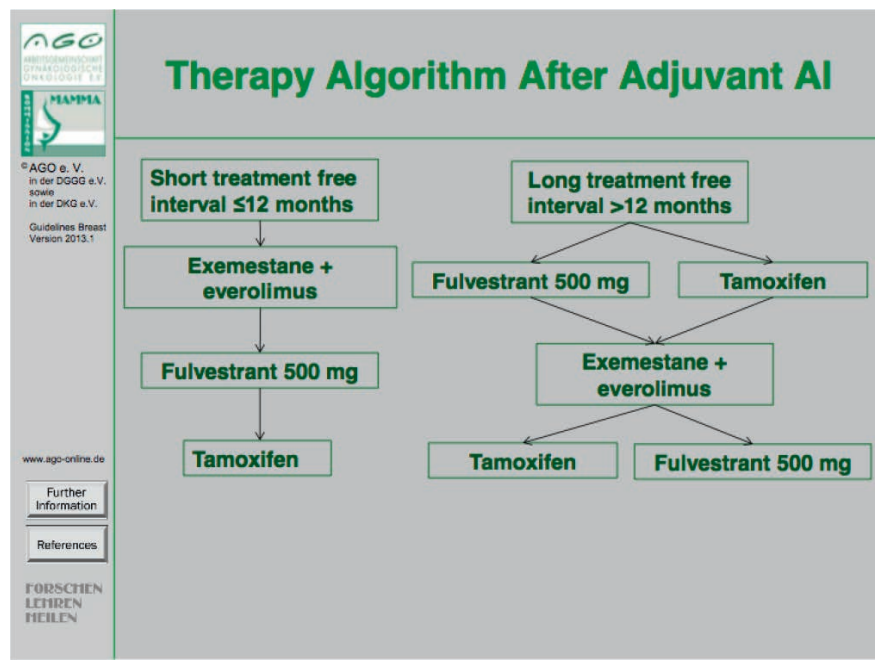

Fig. 2. Therapy algorithm after adjuvant AI treatment. 
crine treatment may be considered, i.e. (i) fulvestrant $(500 \mathrm{mg}$ every 4 weeks) [9] or (ii) a combination of exemestane plus everolimus [10] (fig. 2). In case of disease progression on either one of these two agents at later stages the other agent may be used. Importantly, in selected patients tamoxifen may be given in case of oligosymptomatic disease before chemotherapy is considered.

According to clinical symptoms and endocrine responsiveness, endocrine treatment should be stopped and chemotherapy should be considered for further treatment. However, this should be discussed individually with every patient in shared decision-making.

In HER2-positive HR-positive advanced breast cancer a combination of anastrozol or letrozole and trastuzumab or letrozole and lapatinib can be considered as a choice [11]. However, the progression-free survival (PFS) was quite short in clinical trials. Combination therapies of chemotherapy and HER2-directed treatment should be considered as a more effective option. Again, no significant changes as to the recommendations in this chapter have been considered necessary.

\section{Chemotherapy with or without Targeted Drugs in Metastatic Breast Cancer}

Treatment selection in the advanced situation is based on tumor biology to a similar extent as in the curative setting with the choice of treatment depending on hormone receptor and HER2 status. Other parameters guiding treatment selection are (i) possible combination with compounds of targeted treatment; (ii) previous treatments (and their toxicities); (iii) the aggressiveness of the disease and localization of metastases; (iv) the biologic age; (v) comorbidities (including organ dysfunction); and (vi) the patient's preference and expectations.

The first-line therapy in hormone receptor-positive tumors is generally endocrine therapy. If the leading site of metastasis has been proven to be hormone receptor-negative (preferentially diagnosed by biopsy of at least 1 metastatic lesion) or if the course of the disease suggests endocrine resistance or urgent need of response, cytotoxic chemotherapy is indicated $(\mathrm{LoE} 1 \mathrm{aA}, \mathrm{AGO}++)$. This is in accordance with the recently published First International Consensus Guidelines for Advanced Breast Cancer (ABC 1) [12]. The use of anthracyclines (including liposomal anthracyclines) and taxanes remains state of the art in first-line therapy of metastatic breast cancer. Vinorelbine, capecitabine, and nanoparticle albumin-bound (nab)-paclitaxel are reasonable alternatives. Monotherapy is preferred over polychemotherapy in non-life-threatening situations. Particularly in single-agent therapy, it is recommended to treat as long as the therapeutic index remains positive (LoE $2 \mathrm{bB}, \mathrm{AGO}+$ ). Monitoring of the treatment response should be performed by assessing the tumor burden at baseline and approximately every 2 months, i.e. every $2-4$ cycles of treatment. Assessment of a target lesion may be sufficient. In slow growing disease, longer intervals are acceptable (AGO++). Although some evidence is already available, determination of circulating tumor cells is still considered experimental and recommended preferentially within clinical trials (LoE 1bA, $\mathrm{AGO}+)$.

In some situations, e.g. in TNBC or other aggressive conditions, combination of chemotherapy with bevacizumab may be recommended to increase the response rate and PFS, although not to prolong survival ( $\mathrm{LoE} 2 \mathrm{bB}, \mathrm{AGO}+$ ) [13]. The combination of chemotherapy with other targeted drugs (e.g. sunitinib, sorafinib, vantetanib) is experimental and should not be performed outside of clinical trials. Platinum-based chemotherapy may be useful as further-line treatment, especially in TNBC (LoE 2bB, AGO+/-), even though data from prospective randomized trials are still lacking.

The biggest step forward in the treatment of metastatic breast cancer has been achieved in HER2-overexpressing disease. Pertuzumab in combination with trastuzumab and docetaxel has shown benefit in PFS as well as OS as first-line therapy compared to docetaxel and trastuzumab alone [14] (fig. 3). Furthermore, trastuzumab emtansine (T-DM1) is approved as a further HER2-targeted treatment option in metastatic breast cancer (figs. 4 and 5) and has shown to enhance treatment efficacy (with respect to PFS and OS) in heavily pretreated patients in comparison to lapatinib and capecitabine at favorable toxicity $[15,16]$. Therefore, T-DM1 represents a recommendable treatment option for Patients with HER2-positive metastatic breast cancer in (i) the first line setting diagnosed $\leq 6$ months after adjuvant therapy using taxane and trastuzumab ( $\mathrm{LoE} 2 \mathrm{~b}$ B, AGO +) or (ii) at second line or further $(\mathrm{LoE} 1 \mathrm{bA}, \mathrm{AGO}++)$.

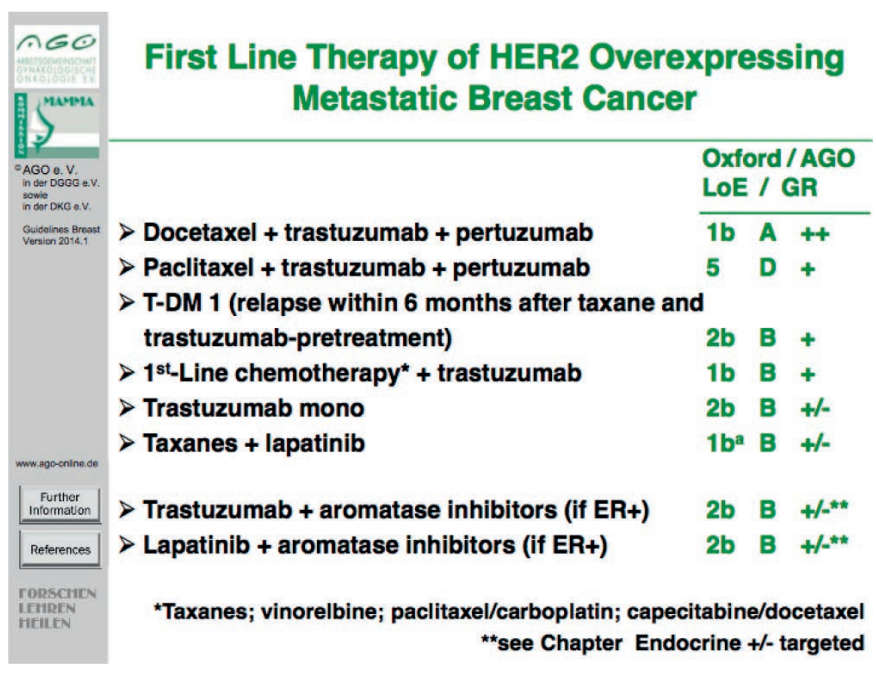

Fig. 3. First-line therapy of HER2-overexpressing metastatic breast cancer. 


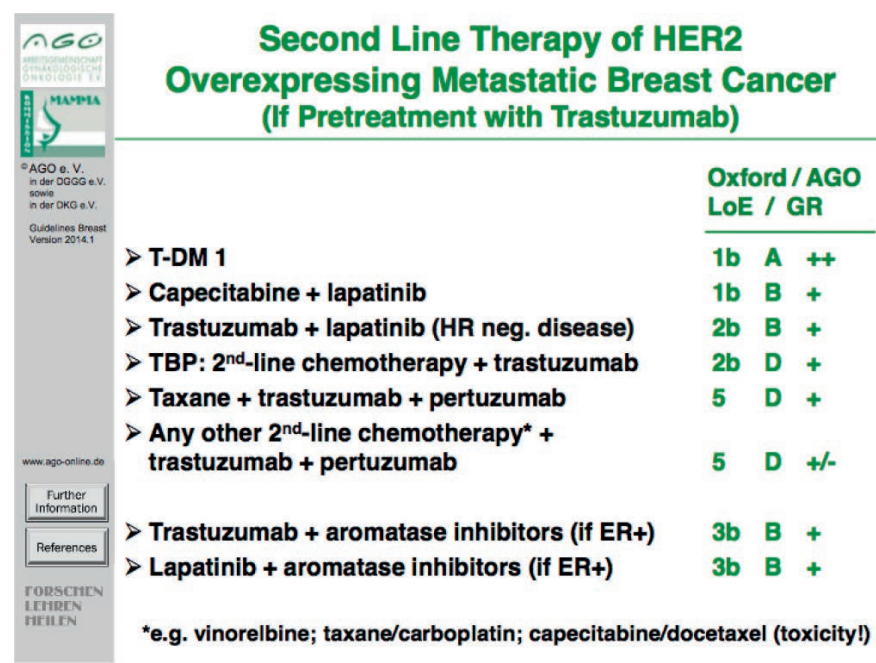

Fig. 4. Second-line therapy of HER2-overexpressing metastatic breast cancer.

\section{Specific Sites of Metastases}

Specific sites of breast cancer metastases are liver, lung, pleura, pericardium, peritoneum, bone marrow, or any soft tissue. Other rare localizations like adrenals, ovaries, uterus, stomach, colon, or placenta have also been reported, however, in such rare cases, controlled trials are not feasible, and treatment options must be discussed individually.

Management of primary stage IV breast cancer focuses primarily on systemic therapy. The impact of the extent of local treatment on patient survival is still a matter of debate. Although some trials suggested an association between local treatment (surgery or radiotherapy) of the primary tumor and prolonged survival, recent reports do not observe these observations $[17,18]$. Therefore, controversy remains as to (i) whether these results reflect a selection of women with good prognosis for primary site therapy; (ii) what fraction of women in published studies were diagnosed with metastatic disease just after surgery, (iii) whether specific subsets of metastases and biological subtypes would derive greater benefit, and (iv) whether local therapy has been performed appropriately with regard to appropriate timing and extent. If surgery of the primary tumor is performed in the metastatic setting, local excision or mastectomy should be done with tumor-free margins $[19,20]$. Axillary surgery is only indicated for bulky disease.

Systemic treatment of metastatic disease is the therapy of choice. Before treatment, metastases should be confirmed by histology to reevaluate diagnosis, HR and HER2 status. Discordance regarding theses markers may occur in about $20 \%$ of patients and may have impact on systemic treatment. If surgery for distant metastases is considered, good overall health, oligometastasis, and a long time between primary treatment and the occurrence of metastases are all favorable factors regarding outcome. Resection of liver metastases may be performed after histological verification if $\mathrm{R} 0$ resection is

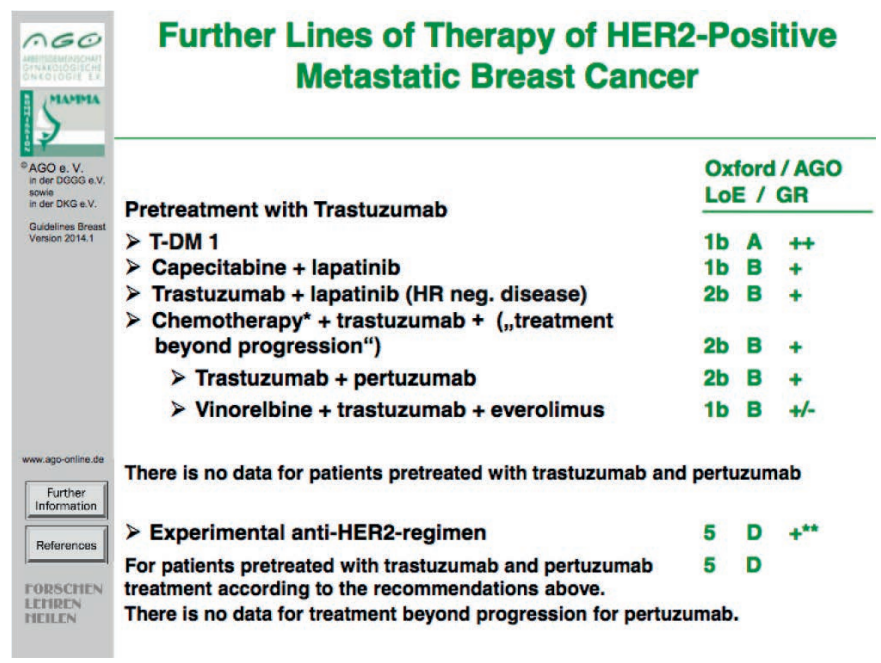

Fig. 5. Further lines of therapy of HER2-positive metastatic breast cancer.

feasible, if no extrahepatic metastases were present, and tumor biology shows a hormone receptor-positive breast cancer responding well to former systemic therapy [21, 22]. Other procedures like regional radiotherapy, thermoablation or chemoembolization are also possible in individual cases $[23,24]$.

For patients with pulmonary metastases, the LoE for a curative approach is low, but some patients might benefit from a metastasectomy followed by an appropriate systemic treatment [25]. In accordance with the treatment of liver metastases, resection of lung metastases should only be performed if R0 resection is feasible and if histological verification was done. The timing of any local intervention may be critical; resection before progression is associated with a better outcome.

About $10 \%$ of all breast cancer patients develop malignant pleural effusion (MPE). In almost 50\% of MPE cases, it is the first sign of metastatic disease, resulting in dyspnea and reduced subjective well-being. It should be treated in symptomatic cases exclusively. Thoracoscopy with talcum pleurodesis (VATS) is the option of choice for MPE. Other sclerosing but more rarely used agents are bleomycin, doxycycline, and mitoxantrone [26]. Continuous pleural drainage with indwelling pleural catheters is a well-tolerated and safe treatment alternative for patients who are not candidates for VATS. Catumaxomab is not yet recommended for MPE.

Overall, $3 \%$ of breast cancer patients will suffer from malignant ascites. Management of ascites takes place in the context of palliative care and aims at improving the quality of life of these patients. Patients with symptomatic ascites should undergo drainage. Local antibody therapy with catumaxomab remains an option in individual cases [27].

Malignant pericardial effusion and cardiac tamponade remain rare metastatic locations in breast cancer patients. In symptomatic patients, drainage and pericardial fenestration are probably the treatment options of choice. For individual 
patients, VATS or ultrasound-guided puncture with instillation of mitoxantrone or bleomycin may be an alternative [28].

The choice between supportive care or specific anticancer treatment for poor-performance status breast cancer patients with multimetastatic disease and pancytopenia due to bone marrow involvement often remains a clinical and human dilemma. Depending on the underlying cancer biology, endocrine therapy or chemotherapy or antibody treatment options should be reconsidered [29]. It has been reported that aggressive combination treatment regimens were effective since most patients showed improved marrow function after chemotherapy, and prolonged survival could be possible. Again, no significant changes to the recommendations in this chapter have been considered necessary.

\section{Central Nervous System Metastases in Breast Cancer}

Breast cancer represents the second most frequent origin of brain metastases (BMs). It is estimated that $10-30 \%$ of patients with metastatic breast cancer are diagnosed with BMs. The incidence of breast cancer BMs is increasing, probably due to the detection of subclinical disease with improved imaging techniques and due to longer control of systemic disease. BMs have become a major cause of morbidity and mortality. Therefore, clinically, BMs are a highly relevant problem. Despite this, only limited data are available for optimal treatment strategies in breast cancer patients, since most studies on BMs involved other tumor entities [30-36]. It is therefore encouraged to register patients with central nervous system (CNS) metastases diagnosed in Germany retrospectively and prospectively in a collaborative registry study. For further information see: www.germanbreastgroup.de.

Several prognostic scores were described for risk estimation in patients with BMs. One of them, the diagnosis-specific graded prognostic assessment (GPA), was published to improve prognostic estimation for patients with BMs. This score was further developed as breast GPA.

The optimal strategy for the treatment of patients with limited (1-3) BMs is unclear. As options, surgery and stereotactic radiotherapy are available for local therapy. The alternative or additional application of whole-brain radiotherapy (WBRT) remains controversial. In general, for patients with limited systemic disease and/or good treatment options, more aggressive treatment is recommended. This is especially true in patients with single BMs where most guidelines recommend combined treatment of surgery/stereotactic radiotherapy and WBRT. The treatment of choice for multiple BMs is WBRT. Compared to single or limited BMs, the role of additional stereotactic radiotherapy is less clear. The recommendations in this chapter have remained largely the same.

\section{Supportive Care}

The granulocyte colony-stimulating factors (G-CSFs), filgrastim and pegfilgrastim, where indicated, are well established. In a comparative effectiveness study, pegfilgrastim prophylaxis was associated with a reduced risk of neutropenia-related or all-cause hospitalization relative to filgrastim prophylaxis [37]. A recent study in high-risk breast cancer demonstrated that $6 \mathrm{mg}$ lipegfilgrastim, a novel glyco-pegylated G-CSF, was as effective as pegfilgrastim in reducing neutropenia in patients with breast cancer receiving myelosuppressive chemotherapy (1b B AGO+) [38]. Concerning prophylaxis of delayed chemotherapy-induced emesis, dexamethasone was not superior to aprepitant but instead had similar efficacy and toxicity in preventing delayed emesis in breast cancer patients treated with anthracycline plus cyclophosphamide chemotherapy and receiving the same antiemetic prophylaxis for acute emesis (1b A AGO++) [39]. Finally, it was acknowledged and confirmed that combined standard oncology care and palliative care should be considered early in the course of illness for patients with metastatic breast cancer and/or high symptom burden [40].

In the last decade, major concerns were raised regarding the use of erythropoiesis-stimulating agents (ESAs) in the treatment of chemotherapy-induced anemia (CIA). Inconsistent results were reported especially in the metastatic setting and by meta-analyses [41-43]. In contrast, no data were available for the adjuvant treatment of breast cancer patients. In the last year, 3 major trials reporting results of the use of ESAs in the adjuvant setting have been published [44-46]. With the exception of the known elevated risk of thrombotic complications, these studies showed no negative impact of the use of ESAs on disease progression or mortality. These results indicate that ESAs are safe drugs for the prevention and treatment of CIA in the adjuvant situation. However, these results are in contradiction to guidelines in Canada and the USA and to the pharmaceutical manufacturers' product labels, which indicate that these agents should not be used when patients receive chemotherapy with curative intent.

\section{Therapy Side Effects}

Acute toxicity and (in most cases) 100-day mortality rates are well documented in the majority of phase III trials. Toxicities are graded according to World Health Organization (WHO) or National Cancer Institute (NCI) standards. Various cytotoxic anticancer drugs have their class-specific toxicity profiles. Anthracycline-based standard chemotherapy regimens in the adjuvant setting demonstrate a relatively low acute toxicity, and treatment-related mortality rates are below $1 \%$. But with respect to long-term side effects, cardiotoxicity is clinically relevant. In addition, the impact of the biological age on adjuvant decision-making has to be considered, e.g. 
by measuring the biological age using the comprehensive geriatric assessment (CGA) [47]. The risk of cardiotoxicity associated with trastuzumab has been reported to be $4 \%$ in monotherapy and $27 \%$ when administered in combination with anthracyclines and cyclophosphamide; however, lifethreatening or severe adverse events are rare [48]. With respect to cardiac toxicity of treatment combinations, see the

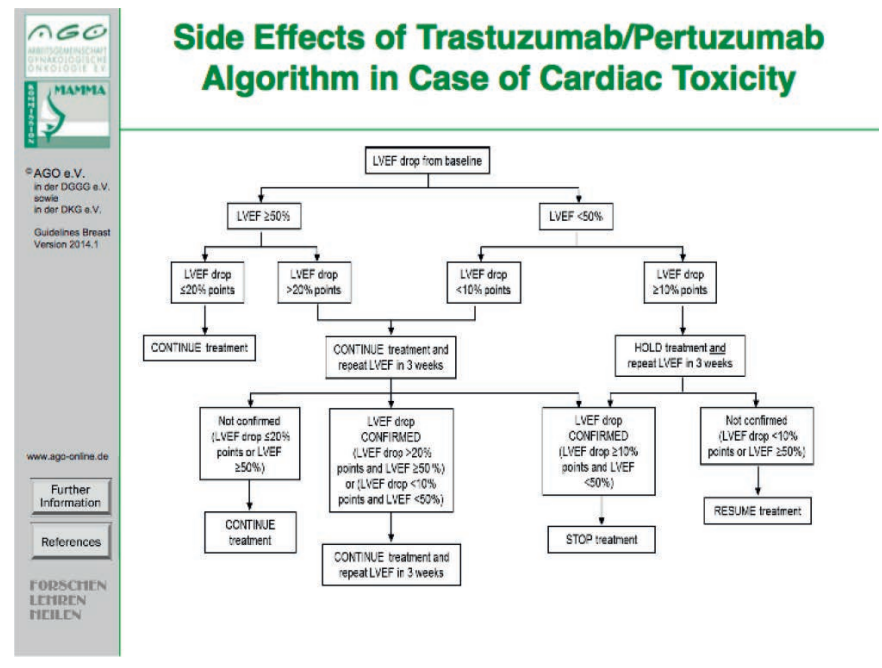

Fig. 6. Side effects of the trastuzumab/pertuzumab algorithm in case of cardiac toxicity. algorithm in figure 6 (based on the Affinity/SAKK 2210 study protocol, SAKK = Schweizerische Arbeitsgemeinschaft für Klinische Krebsforschung, Swiss Oncology Research Network). The combination of pertuzumab and trastuzumab plus docetaxel did not increase the incidence of cardiac adverse events (CLEOPATRA study) [49]. The NeoALTTO study introduced lapatinib and trastuzumab in the neoadjuvant setting together with paclitaxel without major cardiac dysfunction, but a high incidence of diarrhea due to lapatinib was noted [50]. Several neuropsychological studies suggest the association between chemotherapy and long-lasting cognitive deficits, possibly related to therapy-induced structural and functional alterations in the brain [51].

\section{Disclosure Statement}

C.L. has received consultant and speaker honoraria from Amgen, Celgene, Genomic Health, GSK, Novartis, Pierre-Fabre, Roche, and TEVA. M.T. has received consultant and speaker honoraria from AstraZeneca, Amgen, Celgene, Genomic Health, GSK, Novartis, Pierre-Fabre, Serag-Wiessner, Roche, and Sysmex Europe. V.H. has received consultant and speaker honoraria from Novartis and Roche. F.S. has received consultant and speaker honoraria from AstraZeneca, Amgen, Celgene, Novartis, Roche Pharma, and medtronic. The authors have no relevant affiliation or financial involvement with any organization or entity with financial interest in or financial conflict with the subject matter or materials discussed in the manuscript apart from the disclosed.

\section{References}

1 Centre for Evidence Based Medicine: Oxford Level of Evidence, LoE. www.cebm.net.

2 www.ago-online.de/de/fuer-mediziner/leitlinienempfehlungen/.

3 Coleman R; on behalf of the Early Breast Cancer Trialists' Collaborative Group: Effects of Bisphosphonate Treatment on Recurrence and CauseSpecific Mortality in Women With Early Breast Cancer: A Meta-Analysis of Individual Patient Data From Randomised Trials. www.sabcs.org/ PastSymposia/Index.asp\#SABCS2013.

4 Saigal K, Hurley J, Takita C, et al.: Risk factors for locoregional failure in patients with inflammatory breast cancer treated with trimodality therapy. Clin Breast Cancer 2013;13:335-343.

5 Wang J, Xie X, Wang X, et al.: Locoregional and distant recurrences after breast conserving therapy in patients with triple negative breast cancer: a meta-analysis. Surg Oncol 2013;22:247-255.

6 Skinner HD, Strom EA Motwani SB, et al.: Radiation dose escalation for locoregional recurrence of breast cancer after mastectomy. Radiat Oncol 2013;8:13.

7 Jonat W, Kaufmann M, Blamey RW, Howell A, Collins JP, Coates A, Eiermann W, Jänicke F, Njordenskold B, Forbes JF, et al.: A randomised study to compare the effect of the luteinising hormone releasing hormone (LHRH) analogue goserelin with or without tamoxifen in pre- and perimenopausal patients with advanced breast cancer. Eur J Cancer 1995;31A:137-142.

8 Gibson L, Lawrence D, Dawson C, Bliss J: Aromatase inhibitors for treatment of advanced breast cancer in postmenopausal women. Cochrane Database Syst Rev 2009;(4):CD003370.

-9 Di Leo A, Jerusalem G, Petruzelka L, et al.: Results of the CONFIRM phase III trial comparing fulvestrant $250 \mathrm{mg}$ with fulvestrant $500 \mathrm{mg}$ in postmenopausal women with estrogen receptor-positive advanced breast cancer. J Clin Oncol 2010; 28:4594-4600.

10 Baselga J, Campone M, Piccart M, et al.: Everolimus in postmenopausal hormone-receptor-positive advanced breast cancer. N Engl J Med 2012;366: $520-529$.

11 Kaufman B, Mackey JR, Clemens MR, Bapsy PP, Vaid A, Wardley A, Tjulandin S, Jahn M, Lehle M, Feyereislova A, Révil C, Jones A: Trastuzumab plus anastrozole versus anastrozole alone for the treatment of postmenopausal women with human epidermal growth factor receptor 2-positive, hormone receptor-positive metastatic breast cancer: results from the randomized phase III TAnDEM study. J Clin Oncol 2009;27:5529-5537.

12 Cardoso F, Costa A, Norton L, Cameron D, Cufer T, Fallowfield L, Francis P, Gligorov J, Kyriakides S, Lin N, Pagani O, Senkus E, Thomssen C, Aapro M, Bergh J, Di Leo A, El Saghir N, Ganz PA, Gelmon K, Goldhirsch A, Harbeck N, Houssami N, Hudis C, Kaufman B, Leadbeater M, Mayer M, Rodger A, Rugo H, Sacchini V, Sledge G, van't Veer L, Viale G, Krop I, Winer E: 1st international consensus guidelines for advanced breast cancer (ABC 1). Breast 2012;21:242-252.

13 Wagner AD, Thomssen C, Haerting J, Unverzagt S: Vascular-endothelial-growth-factor (VEGF) targeting therapies for endocrine refractory or resistant metastatic breast cancer. Cochrane Database Syst Rev 2012;(7):CD008941.

14 Baselga J, Cortés J, Kim S-B, et al.: Pertuzumab plus trastuzumab plus docetaxel for metastatic breast cancer. N Engl J Med 2012;366:109-119.

15 Verma S, Miles D, Gianni L, et al.: Trastuzumab emtansine for HER2-positive advanced breast cancer. N Engl J Med 2012;367:1783-1791.

16 Hurvitz SA, Dirix L, Kocsis J, et al.: Phase II randomized study of trastuzumab emtansine versus trastuzumab plus docetaxel in patients with human epidermal growth factor receptor 2-positive metastatic breast cancer. J Clin Oncol 2013;31:11571163.

17 Khan SA, Stewart AK, Morrow M: Does aggressive local therapy improve survival in metastatic breast cancer? Surgery 2002;132:620-626.

18 Badwe, R, et al.: Surgical removal of primary tumor and axillary lymph nodes in women with metastatic breast cancer at first presentation: A randomized controlled trial. San Antonio Breast Cancer Symposium 2013; abstr S2-02, www.sabcs. org/pastsymposia/index.asp.

19 Rapiti E, Verkooijen HM, Vlastos G, et al.: Complete excision of primary breast tumor improves survival of patients with metastatic breast cancer at diagnosis. J Clin Oncol 2006;24:2743-2749.

20 Babiera GV, Rao R, Feng L, et al.: Effect of primary tumor extirpation in breast cancer patients who present with stage IV disease and an intact primary tumor. Ann Surg Oncol 2006;13:776782. 
21 Howlader M, Heaton N, Rela M: Resection of liver metastases from breast cancer: towards a management guideline. Int J Surg 2011;9:285-291.

-22 Abbott DE, Brouquet A, Mittendorf EA Andreou A, Meric-Bernstam F, Valero V, Green MC, Kuerer HM, Curley SA, Abdalla EK, Hunt KK, Vauthey JN: Resection of liver metastases from breast cancer: estrogen receptor status and response to chemotherapy before metastasectomy define outcome. Surgery 2012;151:710-716.

- 23 Hoffmann RT, Jakobs TF, Kubisch CH, et al. Radiofrequency ablation after selective interna radiation therapy with yttrium 90 microspheres in metastatic liver disease - is it feasible? Eur J Radiol 2010;74:199-205.

-24 Vogl TJ, Farshid P, Naguib NN, Zangos S: Thermal ablation therapies in patients with breast cancer liver metastases: a review. Eur Radiol 2013;23:797804.

25 Rashid OM, Takabe K: The evolution of the role of surgery in the management of breast cancer lung metastasis. J Thorac Dis 2012;4:420-424.

26 Demmy TL: Optimal management of malignant pleural effusions (results of CALBG 30102). J Natl Compr Canc Netw 2012;10:975-982.

27 Sebastian M, Kuemmel A, Schmidt M, Schmittel A: Catumaxomab: a bispecific trifunctional antibody. Drugs Today (Barc) 2009;45:589-597.

28 Cozzi S, Montanara S, Luraschi A, et al.: Management of neoplastic pericardial effusions. Tumori 2010;96:926-929.

-29 Kopp HG, Krauss K, Fehm T, et al.: Symptomatic bone marrow involvement in breast cancer - clinical presentation, treatment, and prognosis: a single institution review of 22 cases. Anticancer Res 2011:31:4025-4030.

30 Lin NU, Amiri-Kordestani L, Palmieri D, Liewehr DJ, Steeg PS: CNS metastases in breast cancer: old challenge, new frontiers. Clin Cancer Res 2013; 19:6404-6418

-31 Quigley MR, Fukui O, Chew B, Bhatia S, Karlovits $\mathrm{S}$ : The shifting landscape of metastatic breast cancer to the CNS. Neurosurg Rev 2013;36:377-382.

- 32 Sperduto PW, Kased N, Roberge D, Xu Z, Shanley R, Luo X, Sneed PK, Chao ST, Weil RJ, Suh J, Bhatt A, Jensen AW, Brown PD, Shih HA, Kirkpatrick J, Gaspar LE, Fiveash JB, Chiang V, Knisely JP, Sperduto CM, Lin N, Mehta M: Effect of tumor subtype on survival and the graded prognostic assessment for patients with breast cancer and brain metastases. Int J Radiat Oncol Biol Phys 2012;82:2111-2117.

33 Tsao MN, Lloyd N, Wong RK, Chow E, Rakovitch E, Laperriere N, Xu W, Sahgal A: Whole brain radiotherapy for the treatment of newly diagnosed multiple brain metastases. Cochrane Database Syst Rev 2012;(4):CD003869.

34 Tsao MN, Rades D, Wirth A, Lo SS, Danielson BL, Gaspar LE, Sperduto PW, Vogelbaum MA, Radawski JD, Wang JZ, Gillin MT, Mohideen N, Hahn CA, Chang EL: Radiotherapeutic and surgical management for newly diagnosed brain metastasis(es): an American Society for Radiation Oncology evidence-based guideline. Pract Radiat Oncol 2012;2:210-225.

35 Lin NU, Carey LA, Lui MC, et al.: Phase II trial of lapatinib for brain metastases in patients with human epithelial growth factor 2-positive breast cancer. J Clin Oncol 2008;26:1993-1999.

36 Bachelot T, Romieu G, Campone M, Diéras V, Cropet C, Dalenc F, Jimenez M, Le Rhun E, Pierga JY, Gonçalves A, Leheurteur M, Domont J, Gutierrez M, Curé H, Ferrero JM, LabbeDevilliers C: Lapatinib plus capecitabine in patients with previously untreated brain metastases from HER2-positive metastatic breast cancer (LANDSCAPE): a single-group phase 2 study. Lancet Oncol 2013;14:64-71.

37 Naeim A, Henk HJ, Becker L, Chia V, Badre S, Li X, Deeter R: Pegfilgrastim prophylaxis is associated with a lower risk of hospitalization of cancer patients than filgrastim prophylaxis: a retrospective United States claims analysis of granulocyte colony-stimulating factors (G-CSF). BMC Cancer 2013; 13:11.

38 Bondarenko I, Gladkov OA, Elaesser R, Buchner A, Bias P: Efficacy and safety of lipegfilgrastim versus pegfilgrastim: a randomized, multicenter, active-control phase 3 trial in patients with breast cancer receiving doxorubicin/docetaxel chemotherapy. BMC Cancer 2013;13:386.

39 Roila F, Ruggeri B, Ballatori E, Del Favero A, Tonato M: Aprepitant versus dexamethasone for preventing chemotherapy-induced delayed emesis in patients with breast cancer: a randomized double-blind study. J Clin Oncol 2014;32:101-106.

40 Smith TJ, Temin S, Alesi ER, et al.: American Society of Clinical Oncology provisional clinical opinion: the integration of palliative care into standard oncology care. J Clin Oncol 2012;30:880-887.
41 Leyland-Jones B, Semiglazov V, Pawlicki M, et al.: Maintaining normal hemoglobin levels with epoetin alfa in mainly nonanemic patients with metastatic breast cancer receiving first-line chemotherapy: a survival study. J Clin Oncol 2005;23:5960-5972.

42 Tonelli M, Hemmelgarn B, Reimann $\mathrm{T}$, et al.: Benefits and harms of erythropoiesis-stimulating agents for anemia related to cancer. A meta-analysis. CMAJ 2009;180:E62-E71.

43 Glaspy J, Crawford J, Vansteenkiste J, et al.: Erythropoiesis-stimulating agents in oncology: a study-level meta-analysis of survival and other safety outcomes. Br J Cancer 2010;102:301315.

44 Moebus V, Jackisch C, Schneeweiss A, et al.: Adding epoetin alfa to intense dose-dense adjuvant chemotherapy for breast cancer: randomized clinical trial. J Natl Cancer Inst 2013;105:1018-1026.

45 Swain SM, Tang G, Geyer CE, et al.: Definitive results of a phase III adjuvant trial comparing three chemotherapy regimens in women with operable, node-positive breast cancer: the NSABP B-38 trial. J Clin Oncol 2013;31:3197-3204.

46 Nitz U, Gluz O, Oberhoff C, et al.: Final results from the prospective phase III WSG-ARA trial: impact of adjuvant darbepoetin alfa on event-free survival in early breast cancer. Ann Oncol 2014; 25:75-80.

47 Pallis AG, Ring A, Fortpied C, et al.: EORTC workshop on clinical trial methodology in older individuals with a diagnosis of solid tumors. Ann Oncol 2011;22;1922-1926.

48 Keefe DL: Traztuzumab-associated cardiotoxicity. Cancer 2002;95;1592-1600.

49 Swain SM, Ewer MS, Cortés J, Amadori D, Miles D, Knott A, Clark E, Benyunes MC, Ross G, Baselga J: Cardiac tolerability of pertuzumab plus trastuzumab plus docetaxel in patients with HER2positive metastatic breast cancer in CLEOPATRA Oncologist 2013;18:257-264.

50 Baselga J, Bradbury I, Eidtmann H, et al.: Lapatinib with trastuzumab for HER2-positive early breast cancer (NeoALTTO). Lancet 2012;379;633640.

51 Koppelmanns V, Breteler MM, Boogerd W, Seynaeve C, Schagen SB: Late effects of adjuvant chemotherapy for adult onset non-CNS cancer; cognitive impairment, brain structure and risk of dementia. Crit Rev Oncol Hematol 2013;88:87101. 